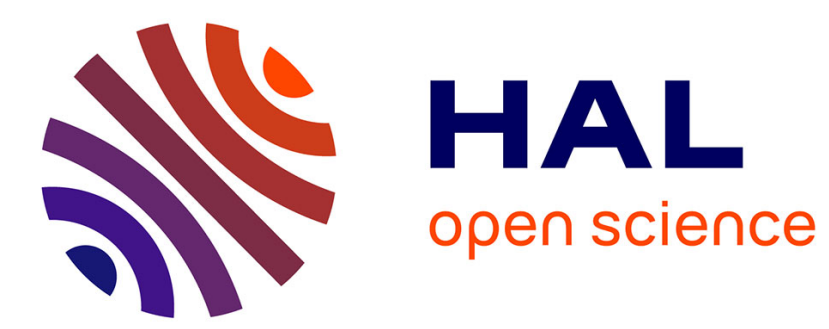

\title{
Locally self-avoiding Eulerian tours
}

Tien-Nam Le

\section{To cite this version:}

Tien-Nam Le. Locally self-avoiding Eulerian tours. Journal of Combinatorial Theory, Series B, 2019, 135, pp.279 - 294. 10.1016/j.jctb.2018.08.008 . hal-03486905

\section{HAL Id: hal-03486905 https://hal.science/hal-03486905}

Submitted on 20 Dec 2021

HAL is a multi-disciplinary open access archive for the deposit and dissemination of scientific research documents, whether they are published or not. The documents may come from teaching and research institutions in France or abroad, or from public or private research centers.
L'archive ouverte pluridisciplinaire HAL, est destinée au dépôt et à la diffusion de documents scientifiques de niveau recherche, publiés ou non, émanant des établissements d'enseignement et de recherche français ou étrangers, des laboratoires publics ou privés.

\section{(ㄷ)(1) $\$$}

Distributed under a Creative Commons Attribution - NonCommercial| 4.0 International 


\title{
Locally self-avoiding eulerian tours
}

\author{
Tien-Nam Le* \\ Laboratoire d'Informatique du Parallélisme \\ École Normale Supérieure de Lyon \\ 69364 Lyon Cedex 07, France
}

\begin{abstract}
It was independently conjectured by Häggkvist in 1989 and Kriesell in 2011 that given a positive integer $\ell$, every simple eulerian graph with high minimum degree (depending on $\ell$ ) admits an eulerian tour such that every segment of length at most $\ell$ of the tour is a path. Bensmail, Harutyunyan, Le and Thomassé recently verified the conjecture for 4-edge-connected eulerian graphs. Building on that proof, we prove here the full statement of the conjecture. This implies a variant of the path case of Barát-Thomassen conjecture that any simple eulerian graph with high minimum degree can be decomposed into paths of fixed length and possibly an additional shorter path.
\end{abstract}

Keywords: eulerian graphs, path-decomposition, Barát-Thomassen conjecture

\section{Introduction}

Unless stated otherwise, graphs considered here are simple and undirected, while multigraphs may contain multiple edges and loops, where each loop contributes two to the degree of the incident vertex.

Given a multigraph $G$, a walk of $G$ is a sequence of alternating vertices and edges $v_{0}, e_{1}, v_{1}, \ldots, v_{\ell-1}, e_{\ell}, v_{\ell}$ of $G$ where each edge $e_{i}=\left\{v_{i-1} v_{i}\right\}$. If $v_{0}=v_{\ell}$, the walk is called a tour. A tour of $G$ is Eulerian if every edge of $G$ appears in the tour exactly once. Given an Eulerian tour $\mathcal{E}$ of $G$, for every positive integer

\footnotetext{
* Corresponding author

Email address: tien-nam.le@ens-lyon.fr (Tien-Nam Le)
}

Preprint submitted to Journal of Combinatorial Theory, Series B 
$\ell$, a walk $v_{0}, e_{1}, v_{1}, \ldots, v_{\ell-1}, e_{\ell}, v_{\ell}$ where any $e_{i}, e_{i+1}$ are consecutive edges of $\mathcal{E}$ is called a segment of length $\ell$ of $\mathcal{E}$. We say that $\mathcal{E}$ is $\ell$-step self-avoiding if every segment of length at most $\ell$ of $\mathcal{E}$ is a path, which is equivalent to that $\mathcal{E}$ "contains" no cycle of length at most $\ell$.

Häggkvist ([7], Problem 3.3) and Kriesell [9] independently conjectured that high minimum degree is a sufficient condition for the existence of an $\ell$-step self-avoiding Eulerian tour.

Conjecture $1.1([7,9])$. For every positive integer $\ell$, there is an integer $d_{\ell}$ such that every Eulerian graph $G$ with minimum degree at least $d_{\ell}$ admits an $\ell$-step self-avoiding Eulerian tour.

Häggkvist also asked to identify the minimum of $d_{\ell}$ if it exists. For the case ${ }_{20} \ell=3$, i.e. triangle-free Eulerian tours, Adelgren [1] characterized all graphs with maximum degree at most 4 which admit a triangle-free Eulerian tour before Oksimets [12] proved Conjecture 1.1 for $\ell=3$ with a sharp bound $d_{3}=6$. Bensmail, Harutyunyan, Le and Thomassé recently verified Conjecture 1.1 for 4-edge-connected Eulerian graphs.

${ }_{25}$ Theorem 1.2 ([4], Theorem 5.1). For every positive integer $\ell$, there is an integer $d_{\ell}^{\prime}$ such that every 4-edge-connected Eulerian graph $G$ with minimum degree at least $d_{\ell}^{\prime}$ admits an $\ell$-step self-avoiding Eulerian tour.

In this paper we verify Conjecture 1.1.

Theorem 1.3. For every positive integer $\ell$, there is an integer $d_{\ell}$ such that every Eulerian graph $G$ with minimum degree at least $d_{\ell}$ admits an $\ell$-step selfavoiding Eulerian tour.

Theorem 1.3 gives an immediate corollary on edge-decomposition of graphs. An edge-decomposition of a graph $G$ consists of edge-disjoint subgraphs whose union is G. Barát and Thomassen in 2006 considered edge-decompositions of 35 graphs into copies of a given tree and conjectured that, together with the necessary condition that $|E(H)|$ divides $|E(G)|$, large edge-connectivity may be an additional sufficient condition. 
Conjecture 1.4 (Barát-Thomassen conjecture, [2]). For any fixed tree $T$, there is an integer $c_{T}$ such that every $c_{T}$-edge-connected graph with number of edges divisible by $|E(T)|$ can be decomposed into subgraphs isomorphic to $T$.

Conjecture 1.4 was recently solved by Bensmail, Harutyunyan, Le, Merker and Thomassé in [3]. For a summary of the progress towards the conjecture, we hence refer the interested reader to that paper. Before that, the path case of the conjecture was verified by Botler, Mota, Oshiro and Wakabayashi in [5], and then was improved by Bensmail, Harutyunyan, Le, and Thomassé [4] that, for path-decompositions, high minimum degree is a sufficient condition provided the graph is 24-edge-connected. Very recently, Klimošová and Thomassé [8] reduced the edge-connectivity condition from 24 to 3, which is known to be sharp (see [4]).

Returning to $\ell$-step self-avoiding Eulerian tours, by cutting the tour found by Theorem 1.3 into paths of length $\ell$, we obtain the following variant of the path case of Barát-Thomassen conjecture.

Corollary 1.5. For every integer $\ell \geq 2$, there is an integer $d_{\ell}$ such that every Eulerian graph with minimum degree at least $d_{\ell}$ can be decomposed into paths of length $\ell$ and possibly an additional path of length less than $\ell$.

Clearly, the theorems above cannot be extended to multigraphs; a multigraph consisting of two vertices linked by many edges is a counterexample. However, the main tool to prove Theorem 1.3 is indeed a weak extension of Theorem 1.2 to multigraphs. Roughly speaking, we only require the Eulerian tour to behave well on a given simple subgraph, not necessary on the whole multigraph.

Theorem 1.6. For every integer $\ell$, there is an integer $d_{\ell}$ such that for every 4-edge-connected Eulerian multigraph $G$ with minimum degree at least $d_{\ell}$ and every simple subgraph $G^{\prime}$ of $G$, the multigraph $G$ admits an Eulerian tour in which every segment of length at most $\ell$ and consisting of only edges of $G^{\prime}$ is a path. 
This paper is organized as follows. We start by recalling some preliminary results in Section 2. Then we use Theorem 1.6 as a black box to prove Theorem 1.3 in Section 3 before proving Theorem 1.6 in the last section.

70

\section{Preliminaries}

In this section we present all the auxiliary results necessary for our proof of Theorem 1.3. Given a multigraph $G$, let $V(G)$ and $E(G)$ denote its vertex and edge sets, respectively. For any subset $X$ of $V(G)$, let $G[X]$ denote the subgraph of $G$ induced by $X$. Given a vertex $v$ of $G$, we denote by $d_{G}(v)$ the degree of $v$ 75 in $G$. Given a subgraph $H=(V, F)$ of a multigraph $G=(V, E)$, we denote by $G \backslash H$ the multigraph $(V, E \backslash F)$.

We start by recalling the definition of cactus graphs. A connected loopless multigraph $G$ is a cactus if every edge belongs to at most one cycle. The singleton graph is a cactus by convention. Clearly, if a cactus is Eulerian then

so every edge belongs to exactly one cycle. The following is a well-known property of cactus graphs.

Proposition 2.1. There are at most two edge-disjoint paths between any two distinct vertices of a cactus.

We also recall three classical results. All of them are originally stated only 85 we state their multigraph version. The first result due to de Werra (cf. [13], Theorem 8.7), asserting that every multigraph has a balanced improper edgecoloring.

Proposition 2.2. Let $G$ be a multigraph and $k \geq 2$ be an integer. There is an 90 improper edge-coloring of $G$ with $k$ colors such that for every vertex $v$ and every pair of colors $i \neq j$, we have $\left|d_{i}(v)-d_{j}(v)\right| \leq 4$, where $d_{i}(v)$ is the number of edges of color $i$ incident with $v$.

The second is a result by Nash-Williams [11] implying that every multigraph with high edge-connectivity admits a balanced orientation with high arc- 
connectivity. In the following, a directed multigraph $D$ is $k$-arc-strong if the removal of any set of at most $k-1$ arcs leaves $D$ strongly-connected, and $d_{D}^{+}(v)$ and $d_{D}^{-}(v)$ denote the outdegree and indegree of $v$ in $D$, respectively.

Proposition 2.3. Every $2 k$-edge-connected multigraph has an orientation $D$ such that $D$ is $k$-arc-strong and $\left|d_{D}^{-}(v)-d_{D}^{+}(v)\right| \leq 1$ for every vertex $v$.

The third result by Edmonds [6] expresses a condition for a directed multigraph to admit many arc-disjoint rooted arborescences. In the statement, an out-arborescence of a directed multigraph $D$ refers to a rooted spanning tree $T$ of $D$ whose arcs are oriented in such a way that the root has indegree 0 , and every other vertex has indegree 1 .

Proposition 2.4. A directed multigraph $D$ has $k$ arc-disjoint out-arborescences rooted at a given vertex $v$ if and only if for any vertex $u \neq v$, there are $k$ arcdisjoint paths from $v$ to $u$.

We close this section with a result by Jackson (cf. [10], Theorem 6.3). Given a loopless multigraph $G$, for every vertex $v$, let $E_{v}$ be the set of edges incident with $v$. A generalized transition system $\mathcal{S}$ of $G$ is a set of functions $\left\{S_{v}\right\}_{v \in V(G)}$ with $S_{v}: E_{v} \rightarrow 2^{E_{v}}$ such that $e_{2} \in S_{v}\left(e_{1}\right)$ whenever $e_{1} \in S_{v}\left(e_{2}\right)$. We say that an Eulerian tour $\mathcal{E}$ is compatible with $\mathcal{S}$ if for any two edges $e_{1}$ and $e_{2}$ such that $e_{1} \in S_{v}\left(e_{2}\right)$ for some $v$, then $e_{1}$ and $e_{2}$ are not consecutive edges of $\mathcal{E}$.

Proposition 2.5. Let $\mathcal{S}$ be a generalized transition system of a loopless Eulerian multigraph $G$ such that $\left|S_{v}(e)\right|=0$ if $d(v)=2$ and $\left|S_{v}(e)\right| \leq d(v) / 2-2$ if $d(v) \geq 4$ for any vertex $v$ and any edge $e$ incident with $v$. Then $G$ admits an Eulerian tour compatible with $\mathcal{S}$.

\section{Proof of Theorem 1.3}

The main idea of the proof of Theorem 1.3 is as follows. We first partition the original graph $G$ into 4-edge-connected Eulerian "induced subgraphs"; these subgraphs are structurally linked by a big cactus. We then apply Theorem 1.6 
to obtain a well-behaved Eulerian tour of each subgraph, and finally connect these tours by the cactus to get an Eulerian tour of $G$.

Given a multigraph $G=(V, E)$, to contract a set of vertices $X \subset V$, we remove all edges inside $X$, and then merge the vertices of $X$ to a new vertex $x$, and each edge incident to $x$ corresponds to an edge incident to some $v \in X$. Note that if the sum of degrees of vertices of $X$ is even, then the degree of $x$ is even.

Let $G=(V, E)$ be an Eulerian multigraph and $\mathcal{X}$ be a partition of $V$ into non-empty sets $X_{1}, X_{2}, \ldots, X_{k}$ for some positive integer $k$. Let $M_{\mathcal{X}}$ be the loopless multigraph obtained from $G$ by contracting each $X_{i}$ to a new vertex $x_{i}$. Clearly, the degree of each $x_{i}$ of $M_{\mathcal{X}}$ is even. If $k \geq 2$, we have that $M_{\mathcal{X}}$ is connected since $G$ is connected, and hence $M_{\mathcal{X}}$ is Eulerian.

Let us suppose for the moment that $M_{\mathcal{X}}$ is a cactus. Thus an edge $e$ of $M_{\mathcal{X}}$ belongs to exactly one cycle in $M_{\mathcal{X}}$. Let $e^{\prime}$ be an edge of the same cycle and incident with $e$. We say that $\left\{e, e^{\prime}\right\}$ is a pair at $x_{i}$, where $x_{i}$ is some endpoint shared by $e$ and $e^{\prime}$. Note that every edge belongs to exactly one pair at each of its endpoints, and hence belongs to exactly two pairs in total. Since each edge $e$ of $M_{\mathcal{X}}$ corresponds to an edge of $G$, we may use $e$ to denote both interchangeably. For every pair $\left\{e, e^{\prime}\right\}$ at some $x_{i}$, each edge has a unique endpoint in $X_{i}$, say $u$ and $u^{\prime}$ respectively. We create a new dummy edge $f=u u^{\prime}$ associated with the pair $\left\{e, e^{\prime}\right\}$ (note that $f$ may be a loop). For every $1 \leq i \leq k$, let $F_{i}$ be the edge set of $G\left[X_{i}\right]$ and $\bar{F}_{i}$ be the set of all dummy edges on $X_{i}$, and let $G_{i}=\left(X_{i}, F_{i} \cup \bar{F}_{i}\right)$. We say that the multigraphs $G_{1}, \ldots, G_{k}$ are inherited from $\mathcal{X}$. Clearly, $d_{G_{i}}(v)=d_{G}(v)$ for every $v \in X_{i}$. The following lemma asserts that there is a partition such that the inherited multigraphs are 4-edge-connected and Eulerian, which are essential conditions to employ Theorem 1.6. For the sake of clarity, we do not consider edge-connectivity of multigraphs on a single vertex.

Lemma 3.1. Given an Eulerian multigraph $G=(V, E)$, there exists a partition $\mathcal{X}$ of $V$ such that $M_{\mathcal{X}}$ is a cactus, and every $G_{i}$ inherited from $\mathcal{X}$ is either a 
single vertex with loops or a 4-edge-connected Eulerian multigraph.

Proof. The proof is by induction on $|V|$. For the case $|V|=2$, let $V=\{u, v\}$.

If $G$ has only two edges between $u$ and $v$, then $\mathcal{X}=\{\{u\},\{v\}\}$; otherwise, $\mathcal{X}=\{\{u, v\}\}$. The lemma holds for $|V|=2$.

For the case $|V|>2$, if $G$ is 4-edge-connected, then $\mathcal{X}=\{V(G)\}$. Otherwise, $G$ contains an edge-cut of size 2, i.e. an edge-cut consisting of two edges. Consider an edge-cut partitioning $V$ into $X_{1}$ and $V^{\prime}$ such that $\left|X_{1}\right|$ is minimum among all possible edge-cuts of size 2 . Let's call the two edges of the cut $u_{1} v_{1}$ and $u_{2} v_{2}$, where $u_{1}, u_{2} \in X_{1}$ and $v_{1}, v_{2} \in V^{\prime}$. We create two dummy edges $f=u_{1} u_{2}$ and $f^{\prime}=v_{1} v_{2}$. Let $\bar{F}_{1}=\{f\}$, and $F_{1}=E\left(G\left[X_{1}\right]\right)$. Let $G_{1}=\left(X_{1}, F_{1} \cup \bar{F}_{1}\right)$ and $G^{\prime}=\left(V^{\prime}, E\left(G\left[V^{\prime}\right]\right) \cup\left\{f^{\prime}\right\}\right)$. There are at least two edge-disjoint paths in $G$ between any two distinct vertices of $X_{1}$. If both paths contains vertices of $V^{\prime}$, then the edge-cut must has size at least 4 , a contradiction. Therefore there is a path in $G\left[X_{1}\right]$ between any two distinct vertices of $X_{1}$. Thus if $\left|X_{1}\right|>1$ then $G_{1}$ is connected, and hence is Eulerian since the degree of every vertex of $G_{1}$ is even. Similarly, $G^{\prime}$ is Eulerian.

Suppose that $G_{1}$ contains an edge-cut of size 2 partitioning $X_{1}$ into $X_{1}^{\prime}$ and $X_{1}^{\prime \prime}$. If $u_{1}$ and $u_{2}$ are in the same partition, say $X_{1}^{\prime}$, then that edge-cut is also an edge-cut of $G$ partitioning $V$ into $X_{1}^{\prime \prime}$ and $V^{\prime} \cup X_{1}^{\prime}$, which contradicts the minimality of $\left|X_{1}\right|$. If $u_{1} \in X_{1}^{\prime}$ and $u_{2} \in X_{1}^{\prime \prime}$ then that edge-cut consists of $f$ and another edge, say $e$. Then $\left\{e, u_{1} v_{1}\right\}$ is an edge-cut of $G$ partitioning $V$ into $X_{1}^{\prime}$ and $V^{\prime} \cup X_{1}^{\prime \prime}$, a contradiction again. It follows that $G_{1}$ contains no edge-cut of size 2 , and so is 4-edge-connected.

Applying induction hypothesis to the Eulerian multigraph $G^{\prime}$ gives a partition of $V^{\prime}$ into $\mathcal{X}^{\prime}=\left\{X_{2}, \ldots, X_{k}\right\}$ such that $M_{\mathcal{X}^{\prime}}$ and $G_{2}, \ldots, G_{k}$ inherited from $\mathcal{X}^{\prime}$ satisfy Lemma 3.1. Let $x_{i} \in M_{\mathcal{X}^{\prime}}$ corresponds to $X_{i}$ for every $2 \leq i \leq k$. Set $\mathcal{X}=\mathcal{X}^{\prime} \cup\left\{X_{1}\right\}$ and construct $M_{\mathcal{X}}$ as follows:

(a) If $v_{1}, v_{2} \in G_{i}$ for some $i$, then $M_{\mathcal{X}}$ is obtained from $M_{\mathcal{X}^{\prime}}$ by adding $x_{1}$ and two parallel edges $x_{1} x_{i}$, corresponding to edges $u_{1} v_{1}$ and $u_{2} v_{2}$ of $G$. Hence there is only one pair at $x_{1}:\left\{u_{1} v_{1}, u_{2} v_{2}\right\}$, and $f$ is its associated 
dummy edge. There is one more pair at $x_{i}$ in $M_{\mathcal{X}}$ comparing with $x_{i}$ in $M_{\mathcal{X}^{\prime}}:\left\{v_{1} u_{1}, v_{2} u_{2}\right\}$, and $f^{\prime}$ is its associated dummy edge.

(b) Otherwise, $v_{1} \in G_{i}$ and $v_{2} \in G_{j}$ for some $i \neq j$. There must be an edge $x_{i} x_{j}$ in $M_{\mathcal{X}^{\prime}}$ corresponding to $f^{\prime}$ in $G^{\prime}$. We obtain $M_{\mathcal{X}}$ from $M_{\mathcal{X}^{\prime}}$ by adding vertex $x_{1}$, edge $x_{1} x_{i}$ corresponding to $u_{1} v_{1}$ and edge $x_{1} x_{j}$ corresponding to $u_{2} v_{2}$ together with deleting the edge $x_{i} x_{j}$ corresponding to $f^{\prime}$. There is only one pair at $x_{1}:\left\{u_{1} v_{1}, u_{2} v_{2}\right\}$, and $f$ is its associated dummy edge. The set of pairs at $x_{i}$ (res. $x_{j}$ ) of $M_{\mathcal{X}}$ are identical to the set of pairs at $x_{i}$ (res. $x_{j}$ ) of $M_{\mathcal{X}^{\prime}}$, except that $v_{1} u_{1}$ (res. $v_{2} u_{2}$ ) replaces $f^{\prime}$ in some pair at $x_{i}\left(\right.$ res. at $\left.x_{j}\right)$.

The multigraphs $G_{2}, \ldots, G_{k}$ inherited from $\mathcal{X}$ in this construction are identical to the multigraphs $G_{2}, \ldots, G_{k}$ inherited from $\mathcal{X}^{\prime}$. By induction hypothesis, for every $i \geq 2$, if $G_{i}$ has more than one vertex then it is 4-edge-connected and Eulerian. Note that $x_{1}$ has degree 2 , and $M_{\mathcal{X}^{\prime}}$ is a cactus, then so is $M_{\mathcal{X}}$. This proves the lemma.

Given an Eulerian tour $\mathcal{E}$ of $G$ and a subset $X$ of $V$, a segment $v_{1} v_{2} \ldots v_{r}(r \geq$ $3)$ of $\mathcal{E}$ is an $X$-boomerang if $v_{1}, v_{r} \in X$ and $v_{2}, \ldots, v_{r-1} \notin X$. A projection of $\mathcal{E}$ on $X$ is an Eulerian tour $\mathcal{E}_{X}$ obtained from $\mathcal{E}$ by replacing every $X$-boomerang, say $v_{1} v_{2} \ldots v_{r}$, by a dummy edge (possibly a loop) between $v_{1}$ and $v_{r}$. If $\mathcal{E}_{X}$ is a projection of $\mathcal{E}$, we say $\mathcal{E}$ and $\mathcal{E}_{X}$ are compatible.

Let $G$ be an Eulerian multigraph and $\mathcal{X}$ be a partition of $G$ together with $M_{\mathcal{X}}$ and inherited $G_{1}, \ldots, G_{k}$ given by Lemma 3.1. For every $i$, let $\mathcal{E}_{i}$ be an arbitrary Eulerian tour of $G_{i}$.

Claim 3.2. There exists an Eulerian tour $\mathcal{E}$ of $G$ compatible with all $\mathcal{E}_{i}$. Furthermore, for every pair $\left\{e, e^{\prime}\right\}$ at some $x_{i}$, there is an $X_{i}$-boomerang of $\mathcal{E}$ starting and ending by $e$ and $e^{\prime}$.

Proof. We reuse all notations in the proof of Lemma 3.1 and proceed by induction on $k$. The claim clearly holds for $k=1$. For $k>1$, recall that by the algorithm in the proof of Lemma 3.1, the Eulerian multigraph $G^{\prime}$ has $k-1$ 
inherited multigraphs identical to $G_{2}, \ldots, G_{k}$ of $G$. Hence, by the induction hypothesis applied on $G^{\prime}$, there exists an Eulerian tour $\mathcal{E}^{\prime}$ of $G^{\prime}$ compatible with all $\mathcal{E}_{i}, i \geq 2$, and for every pair $\left\{e, e^{\prime}\right\}$ at some $x_{i}, i \geq 2$, there is an $X_{i}$-boomerang of $\mathcal{E}^{\prime}$ starting and ending by $e$ and $e^{\prime}$. Note that in cases (a) and (b) of the proof of Lemma 3.1, the only pair at $x_{1}$ is $\left\{u_{1} v_{1}, u_{2} v_{2}\right\}$ associated with $f$. Let $W_{1}$ be the walk obtained from $\mathcal{E}_{1}$ by removing $f$, and $\mathcal{E}$ be the Eulerian tour on $G$ obtained from $\mathcal{E}^{\prime}$ by replacing $f^{\prime}$ by the segment $v_{1} u_{1} W_{1} u_{2} v_{2}$. It is straightforward that, in both cases (a) and (b), the tour $\mathcal{E}$ satisfies Claim 3.2.

Let $\left\{e, e^{\prime}\right\}$ be a pair at some $x_{i}$, and $W$ be the $X_{i}$-boomerang of $\mathcal{E}$ starting and ending by $e$ and $e^{\prime}$. Let $\bar{W}$ be the segment obtained from $\mathcal{E}$ by removing $W$.

Claim 3.3. If $W$ visits a vertex $v \notin X_{i}$, then $\bar{W}$ does not visit $v$.

Proof. Suppose that the claim was false. Let $v \in X_{j}$ for some $j \neq i$. Contracting every $X_{i}$ to $x_{i}$ naturally yields from $W$ and $\bar{W}$ two edge-disjoint walks $W_{\mathcal{X}}$ and ${ }_{225} \bar{W}_{\mathcal{X}}$ in $M_{\mathcal{X}}$, respectively. By following $W_{\mathcal{X}}$ from $x_{i}$ to $x_{j}$ and return to $x_{i}$, and then following $\bar{W}_{\mathcal{X}}$ to $x_{j}$, we obtain three edge-disjoint walks between $x_{i}$ and $x_{j}$, contrary to Proposition 2.1 .

Claim 3.4. If $G$ has minimum degree $d$, then whenever $\mathcal{E}$ leaves $X_{i}$, it takes at least d steps to return to $X_{i}$. Proof. The claim is equivalent to that every $X_{i}$-boomerang $W$ has length at least $d$. Suppose that $W$ visits vertex $v \notin X_{i}$. By Claim 3.3, $W$ must contains all edges incident with $v$, and hence has length at least $d$.

We are ready to prove the main theorem.

Proof of Theorem 1.3. Let $G$ be an Eulerian graph with minimum degree at least $d_{\ell}$, the constant of Theorem 1.6. There is a partition $\mathcal{X}=\left\{X_{1}, \ldots, X_{k}\right\}$ of $V(G)$ together with inherited multigraphs $G_{1}, \ldots, G_{k}$ satisfying Lemma 3.1. 
If $G_{i}$ consists of only one vertex and some loops, let $\mathcal{E}_{i}$ be an arbitrary Eulerian tour of $G_{i}$. Otherwise, Lemma 3.1 asserts that $G_{i}$ is Eulerian, 4-edgeconnected, and $d_{G_{i}}(v)=d_{G}(v) \geq d_{\ell}$ for any $v \in X_{i}$. Also note that $G\left[X_{i}\right]$ is a simple subgraph of $G_{i}$. We thus get, by Theorem 1.6, an Eulerian tour $\mathcal{E}_{i}$ of $G_{i}$ of which every segment of length at most $\ell$ and containing only edges of $G\left[X_{i}\right]$ is a path. Claim 3.2 gives an Eulerian tour $\mathcal{E}$ of $G$ compatible with all $\mathcal{E}_{i}$.

The proof is completed by showing that every segment $W$ of length at most $\ell$ of $\mathcal{E}$ is a path. Suppose that $W=W_{1} e_{1} W_{2} e_{2} \ldots e_{t-1} W_{t}$, where each $W_{s}$ (possibly of length 0) contains only vertices of some $X_{i_{s}}$, and $e_{s}$ is an edge between two distinct sets $X_{i_{s}}$ and $X_{i_{s+1}}$. By Claim 3.4, whenever $\mathcal{E}$ leaves some $X_{i_{s}}$, it takes at least $d_{\ell}>\ell$ steps to return to $X_{i_{s}}$, while the length of $W$ is at most $\ell$. Therefore $X_{i_{s}} \neq X_{i_{r}}$ for every $s \neq r$. Because $\mathcal{E}$ is compatible with $\mathcal{E}_{i_{s}}$, and $W_{s}$ contains only vertices of $X_{i_{s}}$, we have that $W_{s}$ is a segment of $\mathcal{E}_{i_{s}}$. Since $W_{s} \subseteq G\left[X_{i_{s}}\right]$ and has length at most $\ell$, it is a path by Theorem 1.6. This means that $W$ is a path, and the proof is complete.

\section{Proof of Theorem 1.6}

\subsection{Path-collections}

We first recall some notions and results in [4]. Let $G=(V, E)$ be a loopless multigraph. A path-collection $\mathcal{P}$ on $G$ is a set of edge-disjoint paths of $G$. We denote by $U_{\mathcal{P}}=\left(V, E^{\prime}\right)$ the multigraph where $E^{\prime}$ is the set of edges of paths in $\mathcal{P}$. If $U_{\mathcal{P}}=G$ then $\mathcal{P}$ is said to be a path-decomposition of $G$. For convenience, from now on, we say collection for path-collection and decomposition for pathdecomposition.

Let us denote by $H_{\mathcal{P}}=\left(V, E^{\prime \prime}\right)$ the multigraph where each edge $u v \in E^{\prime \prime}$ corresponds to a path between $u$ and $v$ in $\mathcal{P}$ (if $\mathcal{P}$ contains several paths from $u$ to $v$, we have as many edges $\left.u v \in E^{\prime \prime}\right)$. The degree of a vertex $v$ in $\mathcal{P}$, denoted $d_{\mathcal{P}}(v)$, is the degree (with multiplicity) of $v$ in $H_{\mathcal{P}}$, which is also the number of paths in $\mathcal{P}$ with endpoint $v$. 
Two edge-disjoint paths of $G$ sharing an endpoint $v$ are conflicting if they also intersect at some vertex different from $v$. Equivalently, we say that two paths of $\mathcal{P}$ issued from the same vertex are conflicting if the corresponding paths in $U_{\mathcal{P}}$ are conflicting. In general, the paths of a collection can pairwise intersect, and hence we would like to measure how much. For every vertex $v \in V$, let $\mathcal{P}(v)$ be the set of paths in $\mathcal{P}$ containing $v$ as an endpoint. The conflict ratio of $v$ is

$$
\operatorname{conf}_{\mathcal{P}}(v):=\frac{\max _{w \neq v}|\{P \in \mathcal{P}(v): w \in P\}|}{d_{\mathcal{P}}(v)} .
$$

265 $\operatorname{conf}(\mathcal{P}) \leq 1$ since $|\mathcal{P}(v)|=d_{\mathcal{P}}(v)$.

Suppose that we have a decomposition $\mathcal{P}$ of an Eulerian graph $G$ with all paths of length at least $\ell$. Then just by concatenating the paths arbitrarily, we obtain a decomposition of $G$ into several circuits since $G$ is Eulerian. If every two consecutive paths (i.e., they are concatenated) are non-conflicting, then all circuits are $\ell$-step self-avoiding. Theorem 4.1 provides a low conflicting decomposition for this purpose. In order to obtain an $\ell$-step self-avoiding Eulerian tour, it is necessary that the process of concatenating returns a single circuit; this is taken care by Lemma 4.2 .

Theorem 4.1 ([4], Theorem 3.4). Let $\ell$ be a positive integer, and $\varepsilon>0$ suffciently small. There is an integer $L_{\ell, \varepsilon}$ such that for every graph $G$ with minimum degree at least $L_{\ell, \varepsilon}$, there is a decomposition $\mathcal{P}$ of $G$ satisfying:

- The length of every path of $\mathcal{P}$ is either $\ell$ or $\ell+1$.

- $\operatorname{conf}(\mathcal{P}) \leq 1 / 4(\ell+10)$

- $(1-\varepsilon) d_{G}(v) \leq \ell d_{\mathcal{P}}(v) \leq(1+\varepsilon) d_{G}(v)$ for every vertex $v$.

Lemma 4.2 ([4], Lemma 4.1). Every 2-edge-connected loopless multigraph $G$ has a collection $\mathcal{P}$ such that the length of every path in $\mathcal{P}$ is either 1 or 2 , and $H_{\mathcal{P}}$ is a subcubic tree spanning $V(G)$. 


\subsection{F-path-collections}

but more involved. The main difficulty here is that certain arguments on simple graphs in the proof of Theorem 1.2 could not be extended to multigraphs. To overcome these difficulties, we introduce the notions of $F$-paths and $F$-pathcollections.

290 hypotheses of Theorem 1.6, the goal is to find an Eulerian tour $\mathcal{E}$ of $G$ such that every segment of $\mathcal{E}$ of length at most $\ell$ and consisting of only edges of $F$ is a path. To this end, we introduce a relaxation of path, called $F$-path, to depict the characteristics of segments of the tour. Let $G=(V, E)$ be a multigraph and $U_{\mathcal{P}}=G$, then $\mathcal{P}$ is called an $F$-decomposition of $G$. We denote by $H_{\mathcal{P}}=\left(V, E^{\prime \prime}\right)$ the multigraph where each edge (possibly a loop) $u v \in E^{\prime \prime}$ corresponds to an $F$-path between $u$ and $v$ in $\mathcal{P}$. The degree of a vertex $v$ in $\mathcal{P}$, denoted $d_{\mathcal{P}}(v)$, is the degree (with multiplicity, and a loop contributes two) of $v$ in $H_{\mathcal{P}}$.

305 the longest subwalk $v e_{1} v_{1} \ldots e_{s} v_{s}$ (possibly of length 0 ) of $P$ such that $e_{1}, \ldots, e_{s} \in$ $F$. There are several remarks. First, every ray is a path. Second, each $F$-path $P$ has exactly two rays; these rays are identical to $P$ if $P$ is covered, and are edge-disjoint if $P$ is uncovered. Third, if $P$ is a closed (obviously uncovered)

We now would like to measure the conflict between two rays. First, two rays of the same $F$-path are defined as non-conflicting, even if they may intersect at some vertex. Second, two rays $P_{v \mid F}$ and $P_{v \mid F}^{\prime}$ (with $P \neq P^{\prime}$ ) issued from some vertex $v$ are conflicting if $P_{v \mid F}$ and $P_{v \mid F}^{\prime}$ also intersect at some vertex different 
from $v$. For every $v \in V$, let $\mathcal{P}(v)$ be the set of $F$-paths in $\mathcal{P}$ containing $v$ as an endpoint, and $\mathcal{P}(v \mid F)$ be the set of rays from $v$ of $F$-paths in $\mathcal{P}$, where a closed $F$-path with endpoint $v$ contributes two rays. We define the conflict ratio of $v$ in $\mathcal{P}$ as

$$
\operatorname{conf}_{\mathcal{P}}(v \mid F):=\frac{\max _{w \neq v}\left|\left\{P_{v \mid F} \in \mathcal{P}(v \mid F): w \in P_{v \mid F}\right\}\right|}{d_{\mathcal{P}}(v)} .
$$

We denote the conflict ratio of $\mathcal{P}$ by $\operatorname{conf}(\mathcal{P} \mid F):=\max _{v} \operatorname{conf}_{\mathcal{P}}(v \mid F)$. We always have $\operatorname{conf}(\mathcal{P} \mid F) \leq 1$ since $|\mathcal{P}(v \mid F)|=d_{\mathcal{P}}(v)$. Note that, when $\mathcal{P}$ is a collection, the definitions of $\mathcal{P}(v)$ and conflict ratio coincide with the definitions given in the beginning of Section 4.1.

Let us first prove an extension of Theorem 4.1 to $F$-decompositions. By saying a ray of $\mathcal{P}$, we mean a ray of some $F$-path of $\mathcal{P}$.

Lemma 4.3. Let $\ell$ be a positive integer, and $\varepsilon>0$ sufficiently small. There is an integer $L_{\ell, \varepsilon}^{\prime}$ such that for every multigraph $G$ with minimum degree at least $L_{\ell, \varepsilon}^{\prime}$ and every simple subgraph $(V, F)$ of $G$, there is an $F$-decomposition $\mathcal{P}$ of $320 \quad$ satisfying:

- Every ray of $\mathcal{P}$ has length at most $\ell+1$.

- Every covered F-path of $\mathcal{P}$ has length at least $\ell$.

- $\operatorname{conf}(\mathcal{P} \mid F) \leq 1 / 4(\ell+9)$.

- $(1-\varepsilon) d_{G}(v) \leq \ell d_{\mathcal{P}}(v) \leq(1+2 \varepsilon) d_{G}(v)$ for every vertex $v$.

325 Proof. Set $L_{\ell, \varepsilon}^{\prime}=\max \left(L_{\ell, \varepsilon}, 2 \ell / \varepsilon\right)$, where $L_{\ell, \varepsilon}$ is the constant of Theorem 4.1. We call all edges of $\bar{F}=E \backslash F$ dummy (note that a dummy edge may be a loop). The main idea is to replace every dummy edge by a pair of edges linking endpoints of the dummy edge to a big clique in order to obtain a simple graph to apply Theorem 4.1. For every dummy edge $e=v_{e, 1} v_{e, 2}$, we create a set of 330 $L_{\ell, \varepsilon}+1$ new vertices $X_{e}=\left\{x_{e, 1}, \ldots, x_{e, L_{\ell, \varepsilon}+1}\right\}$. Let $E_{e}=\left\{x_{e, i} x_{e, j}: i \neq j\right\} \cup$ $\left\{v_{e, 1} x_{e, 1}, v_{e, 2} x_{e, 2}\right\}$. Let $G^{\prime}$ be the multigraph with vertex set $\bigcup_{e \in \bar{F}} X_{e} \cup V$ and edge set $E^{\prime}=\bigcup_{e \in \bar{F}} E_{e} \cup F$. It is immediate that $G^{\prime}$ is simple and $d_{G}(v)=d_{G^{\prime}}(v)$ 
for every $v \in V$, and so $G^{\prime}$ has minimum degree at least $L$. Therefore $G^{\prime}$ admits a decomposition $\mathcal{P}^{\prime}$ satisfying Theorem 4.1.

For every dummy edge $e$ and every $i=1,2$, let $P_{e, i}^{\prime}$ be the path of $\mathcal{P}^{\prime}$ containing $v_{e, i} x_{e, i}$. We denote by $P_{i, j}$ the longest possible subwalk of $P_{e, i}^{\prime}$ such that $P_{e, i}^{\prime}=\ldots x_{e, i} v_{e, i} P_{e, i} \ldots$ and all vertices of $P_{e, i}$ belong to $V$. If $P_{e, i}$ reach the end of $P_{e, i}^{\prime}$, we call $P_{e, i}$ an end-segment; otherwise, we call it a middle-segment. The reader may see here the similarity between end-segments and rays. Clearly, 340 if $P_{e, i}$ is a middle-segment, then $P_{e, i}^{\prime}=\ldots x_{e, i} v_{e, i} P_{e, i} v_{e^{\prime}, j} x_{e^{\prime}, j} \ldots$ for some dummy edge $e^{\prime}$ and $j \in\{1,2\}$ since $P_{e, i}^{\prime}$ leaves $V$ right after finishing $P_{e, i}$. Note also that the lengths of end-segments and middle-segments are at most $\ell+1$ and possibly 0 .

For every dummy edge $e$ and every $i=1,2$, we remove $X_{e}$ and $E_{e}$, and concatenate $P_{e, i}$ with $e$ at $v_{e, i}$. After this process, we obtain a family of walks, in which each walk lies in one of the following types:

(1) An uncovered $F$-path $P=P_{1} e_{1} P_{2} \ldots e_{t-1} P_{t}$ with dummy edges $e_{1}, \ldots, e_{t-1}$, end-segments $P_{1}$ and $P_{t}$, and middle-segments $P_{2}, \ldots, P_{t-1}$. Note that the two end-segments are the rays of this uncovered $F$-path.

(2) A circuit without endpoint, consisting of middle-segments alternate with dummy edges but no end-segments.

Let $\mathcal{P}_{1}$ be the set of all the walks of Type (1) together with all paths of $\mathcal{P}^{\prime}$ containing only vertices of $V$, and $\mathcal{P}_{2}$ be the set of all circuits of Type (2). Note that $\mathcal{P}_{1}$ is an $F$-collection of $G$, and every edge of $G$ belongs to exactly one $F$-path $\mathcal{P}_{1}$ or one circuit of $\mathcal{P}_{2}$. The method of concatenating ensures that for every $v \in V$, the number of rays from $v$ in $\mathcal{P}_{1}$ is equal to number of paths with endpoint $v$ in $\mathcal{P}^{\prime}$. This gives $d_{\mathcal{P}_{1}}(v)=d_{\mathcal{P}^{\prime}}(v)$. Besides, each ray of $\mathcal{P}_{1}$ is the end-segment of some path of $\mathcal{P}^{\prime}$. Therefore two rays of $\mathcal{P}_{1}$ are conflicting only if their corresponding paths in $\mathcal{P}^{\prime}$ are conflicting. Thus all of the following hold true:

- Every ray of $\mathcal{P}_{1}$ has length at most $\ell+1$, since it is either a path or an 
end-segment of some path of $\mathcal{P}^{\prime}$.

- Every covered $F$-path of $\mathcal{P}_{1}$ has length at least $\ell$, since it is a path of $\mathcal{P}^{\prime}$.

- $\operatorname{conf}_{\mathcal{P}_{1}}(v \mid F) \leq \operatorname{conf}_{\mathcal{P}^{\prime}}(v) \leq 1 / 4(\ell+10)$ for every vertex $v$.

- $(1-\varepsilon) d_{G}(v) \leq \ell d_{\mathcal{P}_{1}}(v) \leq(1+\varepsilon) d_{G}(v)$ for every $v$ since $d_{\mathcal{P}_{1}}(v)=d_{\mathcal{P}^{\prime}}(v)$.

We now turn our attention to $\mathcal{P}_{2}$. Every circuit $C \in \mathcal{P}_{2}$ contains at least one dummy edge. We associate $C$ with some vertex $v$ such that $v$ is the endpoint of some dummy edge of $C$. For every $v \in V$, let $C_{1}, \ldots, C_{t}$ be the circuits (if any) associated with $v$, where every $C_{s}=v e_{s} W_{s} v$ with dummy edge $e_{s}$. Let $\hat{P}_{v}=v e_{1} W_{1} v e_{2} W_{2} \ldots v e_{t} W_{t} v$ be the walk starting and ending at $v$ obtained by concatenating all $C_{s}$ in that fashion. Clearly, $\hat{P}_{v}$ is an uncovered $F$-path, of which one ray is $v$ (length 0 ) and another ray is $W_{t}$, a middle-segment of length at most $\ell+1$. Note that for every $v$, we have at most one such $\hat{P}_{v}$. Let $\hat{\mathcal{P}}_{2}=\left\{\hat{P}_{v}: v \in V\right\}$. Then $\hat{\mathcal{P}}_{2}$ is an $F$-collection of $G$ and $U_{\mathcal{P}_{1}} \cup U_{\hat{\mathcal{P}}_{2}}=G$. Hence ${ }_{375} \mathcal{P}=\mathcal{P}_{1} \cup \hat{\mathcal{P}}_{2}$ is an $F$-decomposition of $G$. Then every ray of $\mathcal{P}$ has length at most $\ell+1$, and every covered $F$-path of $\mathcal{P}$ has length at least $\ell$.

For every $v$, the number of rays from $v$ of $\mathcal{P}$ is at most the number of rays from $v$ of $\mathcal{P}_{1}$ plus two (two rays of $\hat{P}_{v}$ if it exists). Hence $d_{\mathcal{P}_{1}}(v) \leq d_{\mathcal{P}}(v) \leq$ $d_{\mathcal{P}_{1}}(v)+2$, and so by definition of conflict ratio, we have

$$
\begin{aligned}
\operatorname{conf}_{\mathcal{P}}(v \mid F) & \leq \frac{d_{\mathcal{P}_{1}}(v) \operatorname{conf}_{\mathcal{P}_{1}}(v \mid F)+2}{d_{\mathcal{P}}(v)} \\
& \leq \operatorname{conf}_{\mathcal{P}_{1}}(v \mid F)+\frac{2}{d_{\mathcal{P}}(v)} \\
& \leq \frac{1}{4(\ell+10)}+\frac{2}{d_{\mathcal{P}}(v)} \\
& \leq \frac{1}{4(\ell+9)} .
\end{aligned}
$$

Finally, we have $(1-\varepsilon) d_{G}(v) \leq \ell d_{\mathcal{P}_{1}}(v) \leq \ell d_{\mathcal{P}}(v)$. And since $L_{\ell, \varepsilon}^{\prime} \geq 2 \ell / \varepsilon$, we have $\ell d_{\mathcal{P}}(v) \leq \ell\left(d_{\mathcal{P}}(v)+2\right) \leq(1+2 \varepsilon) d_{G}(v)$. The proof is complete.

Lemma 4.3 gives us a good $F$-decomposition $\mathcal{P}$ of $G$. We wish to concatenate the $F$-paths of $\mathcal{P}$ to an Eulerian tour. If $H_{\mathcal{P}}$ has an Eulerian tour, we 
naturally obtain an Eulerian tour of $G$ by replacing each edge of $H_{\mathcal{P}}$ by its corresponding $F$-path of $\mathcal{P}$. Hence the goal is achieving the connectivity of $H_{\mathcal{P}}$, which immediately yields Eulerianity thank to the fact that every vertex of $H_{\mathcal{P}}$ has even degree.

Lemma 4.4. Given a positive integer $\ell$, there is an integer $L_{\ell}^{\prime}$ such that for every 4-edge-connected Eulerian multigraph $G$ with minimum degree at least $100 L_{\ell}^{\prime}$ and every simple subgraph $(V, F)$ of $G$, there is an $F$-decomposition $\mathcal{P}$ of $G$ satisfying:

- Every ray of $\mathcal{P}$ has length at most $\ell+3$.

- Every covered $F$-path of $\mathcal{P}$ has length at least $\ell$.

- $\operatorname{conf}(\mathcal{P} \mid F) \leq 1 / 2(\ell+9)$.

- $H_{\mathcal{P}}$ is Eulerian and spans $V(G)$.

Proof. Let us first outline the proof. We wish to obtain connectivity of $\mathcal{P}$. To this end, we decompose $G$ into a collection $\mathcal{P}_{0}$ satisfying Lemma 4.2 and two $F$-collections $\mathcal{P}_{1}$ and $\mathcal{P}_{2}$ satisfying Lemma 4.3 . Then we use $\mathcal{P}_{0}$, which contains only paths of short length, to extend $F$-paths of $\mathcal{P}_{1}$ obtaining a new $F$-collection $\mathcal{P}_{1}^{\prime}$ such that $H_{\mathcal{P}_{1}^{\prime}}$ is connected. Finally, we merge $\mathcal{P}_{1}$ with $\mathcal{P}_{2}$ to obtain $\mathcal{P}$, which inherits connectivity from $\mathcal{P}_{1}$ and low conflict ratio from $\mathcal{P}_{2}$.

Because $G$ is 4-edge-connected, by Proposition 2.3, there is an orientation $D$ of $G$ such that $D$ is 2 -arc-strong and $\left|d_{D}^{+}(v)-d_{D}^{-}(v)\right| \leq 1$ for every $v$. Applying Proposition 2.4 to $D$ with an arbitrary vertex $z$ gives us two arc-disjoint outarborescences, $T_{1}, T_{2}$, rooted at $z$. Each vertex $v$ has indegree at most 1 in each $T_{i}$ ( $z$ has indegree 0$)$. This gives $d_{T_{1} \cup T_{2}}(v) \leq d_{D}^{+}(v)+2 \leq d_{G}(v) / 2+3$ for every vertex $v$ since $\left|d_{D}^{+}(v)-d_{D}^{-}(v)\right| \leq 1$. Because $T_{1} \cup T_{2}$ is loopless and 2-edge-connected, we obtain a collection $\mathcal{P}_{0}$ on $T_{1} \cup T_{2}$ satisfying Lemma 4.2.

Let $G^{\prime}=G \backslash U_{\mathcal{P}_{0}}$. Then $d_{U_{\mathcal{P}_{0}}}(v) \leq d_{T_{1} \cup T_{2}}(v) \leq d_{G}(v) / 2+3$, and so $G^{\prime}$ has minimum degree at least $100 \ell L_{\ell}^{\prime} / 2-3 \geq 48 \ell L_{\ell}^{\prime}$. By Proposition $2.2, G^{\prime}$ has an improper coloring by $45 \ell$ colors such that $\left|d_{i}(v)-d_{j}(v)\right| \leq 4$ for every vertex $v$ 
and every pair of colors $i \neq j$. Let $G_{1}$ be the subgraph of $G^{\prime}$ with edge set of the first color, and $G_{2}=G^{\prime} \backslash G_{1}$. Thus

$$
d_{G_{1}}(v) \leq \frac{1}{45 \ell-1} d_{G_{2}}(v)+4 \leq \frac{d_{G_{2}}(v)}{40 \ell} .
$$

The minimum degrees of both $G_{1}$ and $G_{2}$ are at least $48 \ell L_{\ell}^{\prime} / 45 \ell-4 \geq L_{\ell}^{\prime}$. Therefore there are $F$-decompositions $\mathcal{P}_{1}$ of $G_{1}$ and $\mathcal{P}_{2}$ of $G_{2}$, both satisfying Lemma 4.3. Hence

$$
d_{\mathcal{P}_{1}}(v) \leq \frac{1+2 \varepsilon}{\ell} d_{G_{1}}(v) \leq \frac{1+2 \varepsilon}{40 \ell^{2}} d_{G_{2}}(v) \leq \frac{1+2 \varepsilon}{40 \ell(1-\varepsilon)} d_{\mathcal{P}_{2}}(v),
$$

for every vertex $v$, with an arbitrary small parameter $\varepsilon$. Set $\varepsilon$ small enough (i.e. set $L_{\ell}^{\prime}$ high enough) such that for every $v$,

$$
d_{\mathcal{P}_{1}}(v) \leq \frac{1}{4(\ell+9)} d_{\mathcal{P}_{2}}(v)-3 .
$$

We now turn our attention to the collection $\mathcal{P}_{0}$ and the subcubic spanning tree $H_{\mathcal{P}_{0}}$. Let us consider $H_{\mathcal{P}_{0}}$ as a tree rooted at an arbitrary vertex $z$. In the following claim, we collect two private $F$-paths in $\mathcal{P}_{1}$ for each path in $\mathcal{P}_{0}$ for the process of concatenating later on.

Claim 4.5. For every path $P \in \mathcal{P}_{0}$ with endpoints say $u, v$ where $v$ is the parent of $u$ in $H_{\mathcal{P}_{0}}$, there are two $F$-paths of $\mathcal{P}_{1}(v)$, named $g_{1}(P)$ and $g_{2}(P)$, such that their rays from $v$ do not conflict with $P$ (if $g_{i}(P)$ is closed, one of its rays satisfying that condition is sufficient). Furthermore, $g_{i}(P) \neq g_{j}\left(P^{\prime}\right)$ for any $(i, P) \neq\left(j, P^{\prime}\right)$.

Proof. We first apply Proposition 2.3 to have an orientation $D$ of $H_{\mathcal{P}_{1}}$ such that $\left|d_{D}^{-}(v)-d_{D}^{+}(v)\right| \leq 1$. This orientation yields a natural orientation of $F$-paths of $\mathcal{P}_{1}$. We denote by $\mathcal{P}_{1}^{+}(v \mid F)$ the set of rays of $\mathcal{P}$ leaving $v$ with respect to $D$. Note that each closed $F$-path at $v$ contributes with exactly one ray to $\mathcal{P}_{1}^{+}(v \mid F)$. This gives $\left|\mathcal{P}_{1}^{+}(v \mid F)\right| \geq d_{\mathcal{P}_{1}}(v) / 2-1$.

Since $H_{\mathcal{P}_{0}}$ is subcubic, there are at most 3 paths of $\mathcal{P}_{0}$ with endpoint $v$, say $P_{s}$ for $1 \leq s \leq 3$. Note that each $P_{s}$ has length at most 2 , and so they are incident 
with at most 6 vertices except $v$ in total. Recall that $\operatorname{conf}_{\mathcal{P}_{1}}(v \mid F) \leq 1 / 4(\ell+9)$. For each vertex $w$ among these 6 possible vertices, we have

$$
\left|\left\{P_{v \mid F} \in \mathcal{P}_{1}(v \mid F): w \in P_{v \mid F}\right\}\right| \leq \frac{d_{\mathcal{P}_{1}}(v)}{4(\ell+9)} \leq \frac{2\left|\mathcal{P}_{1}^{+}(v \mid F)\right|+2}{4(\ell+9)} \leq \frac{\left|\mathcal{P}_{1}^{+}(v \mid F)\right|}{12} .
$$

Hence in total there are at most $\left|\mathcal{P}_{1}^{+}(v \mid F)\right| / 2$ rays of $\mathcal{P}_{1}^{+}(v \mid F)$ conflicting with some $P_{s}$. This guarantees that there are at least half of rays in $\mathcal{P}_{1}^{+}(v)$ nonconflicting with all $P_{s}$. We just pick 6 rays among them, and name the $F$-paths of these rays $g_{i}\left(P_{s}\right)$ arbitrarily (these $F$-paths are clearly pairwise distinct). Note also that $\mathcal{P}_{1}^{+}(v) \cap \mathcal{P}_{1}^{+}\left(v^{\prime}\right)=\emptyset$ for any $v \neq v^{\prime}$, so $g_{i}(P) \neq g_{j}\left(P^{\prime}\right)$ for any $(i, P) \neq\left(j, P^{\prime}\right)$.

We can now obtain the connectivity of $H_{1}$ by concatenating each $P$ of $\mathcal{P}_{0}$ to either $g_{1}(P)$ or $g_{2}(P)$. Let us call $\mathcal{T}$ a rooted tree on vertex set $\left\{Y_{1}, Y_{2}, \ldots, Y_{t}\right\}$, where $\left\{Y_{1}, Y_{2}, \ldots, Y_{t}\right\}$ is some partition of $V$ with the following properties:

(A) For every edge $Y_{i} Y_{j}$ of $\mathcal{T}$, there is a corresponding path $v_{i} \ldots v_{j} \in \mathcal{P}_{0}$, where $v_{i} \in Y_{i}$ and $v_{j} \in Y_{j}$.

(B) For every $Y_{i}$, there is an $F$-collection $\mathcal{R}_{i}$ such that $H_{\mathcal{R}_{i}}$ is connected and spans $Y_{i}$, and each $F$-path in $\mathcal{R}_{i}$ is either $g_{1}(P)$ or the concatenation of $P$ and $g_{1}(P)$ for some $P \in \mathcal{P}_{0}$ (if $Y_{i}$ contains a single vertex then $\mathcal{R}_{i}$ is empty).

Such structured-tree $\mathcal{T}$ clearly exists by choosing $\mathcal{T}$ equal to $H_{\mathcal{P}_{0}}$ rooted at $z$, in which each $Y_{i}$ contains a single vertex, and each $\mathcal{R}_{i}$ is empty. Our goal is to repeatedly merge vertices of $\mathcal{T}$ until $\mathcal{T}$ is the singleton graph, which completes the process of concatenating. We consider a leaf $Y_{i}$ of $\mathcal{T}$ with parent $Y_{j}$, corresponding to path $P=v_{i} \ldots v_{j}$ of $\mathcal{P}_{0}$ with $v_{i} \in Y_{i}$ and $v_{j} \in Y_{j}$. Suppose that $g_{1}(P)=v_{j} \ldots y$ and $g_{2}(P)=v_{j} \ldots z$.

- If $y \in Y_{k}$ for some $k \neq i$, we concatenate $P$ and $g_{1}(P)$ at $v_{j}$ and get a $F$-path $P^{*}$. Then we merge $Y_{i}$ into $Y_{k}$ to form new set $Y_{i k}$ (inheriting the position of $Y_{k}$ in tree $\mathcal{T}$ ). Let $\mathcal{R}_{i k}=\mathcal{R}_{i} \cup \mathcal{R}_{k} \cup\left\{P^{*}\right\}$. Since $P^{*}$ connects two vertices of $\mathcal{R}_{i}$ and $\mathcal{R}_{k}$, we have that $H_{\mathcal{R}_{i k}}$ is connected and spans $Y_{i k}$. 
- If $y \in Y_{i}$, we merge $Y_{i}$ to $Y_{j}$ to form new set $Y_{i j}$ (inheriting the position of $Y_{j}$ in tree $\left.\mathcal{T}\right)$. Set $\mathcal{R}_{i j}=\mathcal{R}_{i} \cup \mathcal{R}_{j} \cup\left\{g_{1}(P)\right\}$. Since $g_{1}(P)$ connects two vertices of $\mathcal{R}_{i}$ and $\mathcal{R}_{j}$, we have that $H_{\mathcal{R}_{i j}}$ is connected and spans $Y_{i j}$. We also concatenate $P$ with $g_{2}(P)$ at $v_{j}$ to get another $F$-path and put it back into $\mathcal{P}_{1}$.

The number of vertices of $\mathcal{T}$ is reduced by 1 after each step, while $\mathcal{T}$ still satisfies both properties. Once the process is complete, we end up with a singleton $\mathcal{T}$ and an $F$-collection $\mathcal{R}$ such that $H_{\mathcal{R}}$ is connected and spans $V$. Note that $\mathcal{P}_{0}$ is empty at the end of the process, since exactly one path of $\mathcal{P}_{0}$ is used at each step. We merge $\mathcal{R}$ with $\mathcal{P}_{1}$ to obtain a new collection $\mathcal{P}_{1}^{\prime}$. Consequently, $H_{\mathcal{P}_{1}^{\prime}}$ is connected.

Let $\mathcal{P}=\mathcal{P}_{1}^{\prime} \cup \mathcal{P}_{2}$. Note that $U_{\mathcal{P}}=U_{\mathcal{P}_{1}^{\prime}} \cup U_{\mathcal{P}_{2}}=G$, so $\mathcal{P}$ is an $F$-decomposition of $G$ and $H_{\mathcal{P}}$ is connected. The degrees of all vertices of $G$ are even, then so are the degrees of vertices of $H_{\mathcal{P}}$, and hence $H_{\mathcal{P}}$ is Eulerian. The process of concatenating also ensures that every ray of $\mathcal{P}$ has length at most $\ell+3$ and that every covered $F$-path of $\mathcal{P}$ has length at least $\ell$.

It remains to prove that $\operatorname{conf}(\mathcal{P} \mid F) \leq 1 / 2(\ell+9)$. In the following, by saying $\mathcal{P}_{0}$ or $\mathcal{P}_{1}$, we mean the collection before the process of concatenating. Recall that $H_{\mathcal{P}_{0}}$ is subcubic, so for every vertex $v$, the number of $F$-paths with endpoint $v$ in $\mathcal{P}_{1}^{\prime}$ is at most the number $F$-paths with endpoint $v$ in $\mathcal{P}_{1}$ plus 3 . Combining with (1) yields $d_{\mathcal{P}_{1}^{\prime}}(v) \leq d_{\mathcal{P}_{1}}(v)+3 \leq d_{\mathcal{P}_{2}}(v) / 4(\ell+9)$. Recall that $\operatorname{conf}_{\mathcal{P}_{1}^{\prime}}(v \mid F) \leq 1$ and $\operatorname{conf}_{\mathcal{P}_{2}}(v \mid F) \leq 1 / 4(\ell+9)$. Hence for every vertex $v$, by definition of conflict ratio we have

$$
\begin{aligned}
\operatorname{conf}_{\mathcal{P}}(v \mid F) & \leq \frac{d_{\mathcal{P}_{2}}(v) \operatorname{conf}_{\mathcal{P}_{2}}(v \mid F)+d_{\mathcal{P}_{1}^{\prime}}(v) \operatorname{conf}_{\mathcal{P}_{1}^{\prime}}(v \mid F)}{d_{\mathcal{P}_{2}}(v)+d_{\mathcal{P}_{1}}(v)} \\
& <\operatorname{conf}_{\mathcal{P}_{2}}(v \mid F)+\frac{d_{\mathcal{P}_{1}^{\prime}}(v) \operatorname{conf}_{\mathcal{P}_{1}^{\prime}}(v \mid F)}{d_{\mathcal{P}_{2}}(v)} \\
& \leq \frac{1}{4(\ell+9)}+\frac{1}{4(\ell+9)} \\
& \leq \frac{1}{2(\ell+9)} .
\end{aligned}
$$

This implies $\operatorname{conf}(\mathcal{P} \mid F) \leq 1 / 2(\ell+9)$, and the lemma follows. 
The final step is concatenating $F$-paths of $\mathcal{P}$ to obtain a well-behaved Euleof $F$. It remains to prove that $W$ is a path. Let $P_{1}, P_{2} \ldots, P_{r}$ be consecutive (with respect to $\mathcal{E}$ ) $F$-paths of $\mathcal{P}$ such that $W$ is a subwalk of $P_{1} P_{2} \ldots P_{r}$ and

Proof of Theorem 1.6. Let $d_{\ell}=100 \ell L_{\ell, \varepsilon}^{\prime}$ and $G^{\prime}=(V, F)$. We first obtain an $F$ decomposition $\mathcal{P}$ of $G$ satisfying Lemma 4.4. For every ray $P_{v \mid F}$ of $\mathcal{P}$, each vertex $w \in P_{v \mid F}$ is a conflict point between $P_{v \mid F}$ and at most $d_{\mathcal{P}}(v) / 2(\ell+9)$ other rays. Hence the number of rays conflicting with $P_{v \mid F}$ is at most $(\ell+3) d_{\mathcal{P}}(v) / 2(\ell+9) \leq$ $d_{\mathcal{P}}(v) / 2-2$ since $P_{v \mid F}$ has length at most $\ell+3$.

We wish to apply Proposition 2.5 to $H_{\mathcal{P}}$. Therefore the task now is to eliminate all loops of $H_{\mathcal{P}}$. Let $H_{\mathcal{P}}^{*}$ be the loopless multigraph obtained from $H_{\mathcal{P}}$ by subdividing every loop $e=v v$ into $v x_{e}$ and $x_{e} v$ by a new vertex $x_{e}$. We associate each $v x_{e}$ and $x_{e} v$ with a ray of $P$, where $P \in \mathcal{P}(v)$ is the corresponding $F$-path of $e$.

For every pair of incident vertex-edge $(v, e)$ of $H_{\mathcal{P}}^{*}$, let $S_{v}(e)$ be the set of all edges of $H_{\mathcal{P}}^{*}$ corresponding to rays conflicting with $P_{v \mid F}$, where $P_{v \mid F}$ is ray of $\mathcal{P}$ corresponding to $e$. Since two rays of the same $F$-path are non-conflicting, we have $\left|S_{x_{e}}(e)\right|=0$ for every loop $e$ of $H_{\mathcal{P}}^{*}$. Hence $\left|S_{v}(e)\right| \leq d_{H_{\mathcal{P}}^{*}}(v) / 2-2$ if $d_{H_{\mathcal{P}}^{*}}(v) \geq 4$ and $\left|S_{v}(e)\right|=0$ if $d_{H_{\mathcal{P}}^{*}}(v)=2$ for every pair of incident vertex-edge $(v, e)$ of $H_{\mathcal{P}}^{*}$.

Let $\mathcal{S}=\left\{S_{v}\right\}_{v \in V}$, then $\mathcal{S}$ is a generalized transition system of $H_{\mathcal{P}}^{*}$. Proposition 2.5 asserts that $H_{\mathcal{P}}^{*}$ admits an Eulerian tour $\mathcal{E}_{H_{\mathcal{P}}^{*}}$ compatible with $\mathcal{S}$, i.e., the corresponding rays of any two consecutive edges of $\mathcal{E}_{H_{\mathcal{P}}^{*}}$ are non-conflicting. Clearly, $v x_{e}$ and $x_{e} v$ are two consecutive edges of $\mathcal{E}_{H_{\mathcal{P}}^{*}}$ since $x_{e}$ has degree 2. We therefore naturally obtain from $\mathcal{E}_{H_{\mathcal{P}}^{*}}$ an Eulerian tour $\mathcal{E}_{H_{\mathcal{P}}}$ of $H_{\mathcal{P}}$ by replacing $e$ to the segment $v x_{e} v$ for every loop $e=v v$ of $H_{\mathcal{P}}$. Hence we naturally obtain from $\mathcal{E}_{H_{\mathcal{P}}}$ an Eulerian tour $\mathcal{E}$ of $G$ by replacing every edge of $\mathcal{E}_{H_{\mathcal{P}}}$ by its corresponding $F$-path of $\mathcal{P}$. Note that every two consecutive (with respect to $\mathcal{E})$ rays of $\mathcal{P}$ are non-conflicting.

Let $W$ be a segment of $\mathcal{E}$ of length at most $\ell$ and consisting of only edges 
$W \cap P_{1}, W \cap P_{r} \neq \emptyset$. If $r \geq 3$ then $W$ must contain entirely $P_{2}$. All edges of $W$ belong to $F$, then so does $P_{2}$. Hence $P_{2}$ is a covered $F$-path of length at most $\ell-2$, contrary to the fact that every covered $F$-path of $\mathcal{P}$ has length at least $\ell$. If $r=2$, note that the rays from $v$ of $P_{1}$ and $P_{2}$ are non-conflicting, and $W$ is a subwalk of the concatenation of these two rays. Hence $W$ is a path. If $r=1$ then clearly $W$ is a path, the desired conclusion.

\section{Acknowledgement}

We would like to thank Stéphan Thomassé for valuable discussions, and the reviewer for helpful comments and suggestions.

\section{References}

[1] T. Adelgren. Triangle-free Eulerian tours in graphs with maximum degree at most 4. Discrete Mathematics, 138 (1995) 5-14.

[2] J. Barát and C. Thomassen. Claw-decompositions and Tutte-orientations. Journal of Graph Theory, 52 (2006) 135-146.

[3] J. Bensmail, A. Harutyunyan, T.-N. Le, M. Merker and S. Thomassé. A proof of the Barát-Thomassen conjecture. Journal of Combinatorial Theory, Series B, 124 (2017) 39-55.

[4] J. Bensmail, A. Harutyunyan, T.-N. Le and S. Thomassé. Edge-partitioning a graph into paths: beyond the Barát-Thomassen conjecture. Combinatorica, (2018) 1-25.

[5] F. Botler, G.O. Mota, M. Oshiro and Y. Wakabayashi. Decomposing highly edge-connected graphs into paths of any given length. Journal of Combinatorial Theory, Series B, 122 (2017) 508-542.

[6] J. Edmonds, Edge-disjoint branchings. B. Rustin, editor, Combinatorial Algorithms, Academic Press (1973), 91-96. 
[7] R. Häggkvist. Decompositions of regular bipartite graphs. J. Siemons, editor, Surveys in Combinatoris, pp. Cambridge University Press (1989) 115147.

[8] T. Klimošová and S. Thomassé, Decomposing graphs into paths and trees. Electronic Notes in Discrete Mathematics 61 (2017): 751-757.

[9] M. Kriesell. Fourth international conference on combinatorics, graph theory, and applications, Elgersburg (2011).

[10] B. Jackson, On circuit covers, circuit decompositions and Euler tours of graph, Surveys in Combinatorics, London Mathematical Society Lecture Note Series, 187 (1993) 191-210.

[11] C.St.J.A. Nash-Williams. On orientations, connectivity and odd-vertexpairings in finite graphs. Canadian Journal of Mathematics, 12 (1960) $555-567$.

[12] N. Oksimets. Triangle-free Eulerian tours in graphs with minimum degree at least 6. Licentiat Thesis, Umeå University (1997).

[13] M. Stiebitz, D. Scheide, B. Toft, L. M. Favrholdt. Graph Edge Coloring: Vizing's Theorem and Goldberg's Conjecture. Wiley (2012). 